\title{
Bilateral Neural Control of Visually Guided Hand Movement
}

\author{
Xiaofeng $\operatorname{Lu}^{1,2^{*}}$ \\ ${ }^{1}$ Brain Sciences Center, Veterans Administration Medical Center, Minneapolis, Minnesota 55417, USA \\ ${ }^{2}$ Department of Neurology, School of Medicine, University of Minnesota, Minneapolis, Minnesota 55414, USA
}

Does the right cerebral cortex stay inactive when one slams down on the brake pedal with their right foot in order to stop a car as quickly as possible in an emergency situation? I think not. This is obviously against a common view that the neural control of movement was unilateral: cerebral control, contralateral; cerebellar control, ipsilateral $[1,2]$. Here we discuss our previous studies of temporary inactivation experiments in the motor cortex and cerebellar nucleus of the nonhuman primates, and hypothesize that neural control of initiation of visually guided hand movement is bilateral.

In our previous studies, tow monkeys were trained to practice a visually guided hand reaching task. After the monkeys mastered the task, separate muscimol injections were made into cerebellar Dentate Nucleus (DN) or primary motor cortex (M1) to inactivate the local neural activity during performance of the task. After unilateral injections made into the $\mathrm{DN}$ or the $\mathrm{M} 1$, significantly delayed initiation of hand movement (reaction time, from Target-on/Go-signal to hand release from the home key) was observed whether the monkeys used either hand to perform the task. In contrast, significantly slowed hand movement (movement time, from hand release of the home key to reaching the target) was observed only when the monkeys used the hand ipsilateral to the injected DN or contralateral to the injected M1. These results from the two monkeys were consistent. Our data indicate that both the DN and the M1 bilaterally contribute to reaction time, and unilaterally to movement time. This furthers the hypothesis that motor control in the brain is bilateral rather than unilateral.

This theory has been supported by several independent studies. Examples include that motor response was delayed bilaterally by the unilateral frontal cortex lesion [3]; and clinical studies reported that the reaction time exceeded the normal range bilaterally after unilateral excisions in the supplementary motor area [4]. In addition, both the DN and the M1 contain diverse capabilities in relation to cognitive aspect of motor control during visually guided hand movement [5-7]. Thus, bilateral involvement of the $\mathrm{DN}$ and the M1 may be not surprising. To initiate movement, contribution of neurons from relatively wider areas of the brain, even across both hemispheres of the brain is often required.

In the future, analysis of potential neural correlation with the bilateral effects of the reaction time may need to be done in both sides of the brain, particularly during the pre-motor period. This will help to understand how the single neurons are involved in the movement bilaterally. Last, clinical frontline also can benefit from this new theory of bilateral neural control. The questions of how to incorporate this new theory of bilateral neural control of movement into the clinical examination or evaluation, and how to create new therapies during the rehabilitation, in particular, to patients with hemiplegia soon need to be answered.

\section{References}

1. Day BL, Brown $P$ (2001) Evidence for subcortical involvement in the visual control of human reaching. Brain 124: 1832-1840.

2. Döbrössy MD, Dunnett SB (1997) Unilateral striatal lesions impair response execution on a lateralised choice reaction time task. Behav Brain Res 87: 159171.

3. Kazennikov O, Hyland B, Wicki U, Perrig S, Rouiller EM, et al. (1998) Effects of lesions in the mesial frontal cortex on bimanual co-ordination in monkeys. Neuroscience 85: 703-716.

4. Bell KR, Traylor GH, Anderson ME, Berger MS, Ojemann GA (1994) Features of targeted arm movement after unilateral excisions that included the supplementary motor area in humans. Brain Res 655: 202-212.

5. Lu X, Miyachi S, Takada M (2012) Anatomical evidence for the involvement of medial cerebellar output from the interpositus nuclei in cognitive functions. Proc Natl Acad Sci U S A 109: 18980-18984.

6. Voogd J, Ruigrok TJH (2012) Cerebellum and precerebellar nuclei. The Human Nervous System (3rd edn), Elsevier, Amsterdam.

7. Lu X (2013) The Cerebellum and cerebello-thalamuo-cortical channels contribute to new learning and long-term memory of motor skill. Brain Disord Ther 2: e106.

\footnotetext{
*Corresponding author: Xiaofeng Lu, Research Physiologist, VA Medical Center, Minneapolis, USA, Tel: 612-467-4583; E-mail: luxxx049@umn.edu

Received January 29, 2014; Accepted January 31, 2014; Published February 3, 2014

Citation: Lu X (2014) Bilateral Neural Control of Visually Guided Hand Movement Brain Disord Ther 3:e110. doi:10.4172/2168-975X.1000e110

Copyright: (c) $2014 \mathrm{Lu} \mathrm{X}$. This is an open-access article distributed under the terms of the Creative Commons Attribution License, which permits unrestricted use, distribution, and reproduction in any medium, provided the original author and source are credited.
} 